\title{
DATA
}

\section{Forest resources in China}

\section{Figures and Graphics}

From the $8^{\text {th }}$ and latest National Forest Inventory by the State Forestry Administration of China

\section{Highlights of forest resources in China}

- Forest area: 208 million $\mathrm{hm}^{2}$

- Forest coverage: $21.63 \%$

- Forest stock volume: 15.137 billion $\mathrm{m}^{3}$

\section{Data}

2.1 Forest area of the top six countries in the world

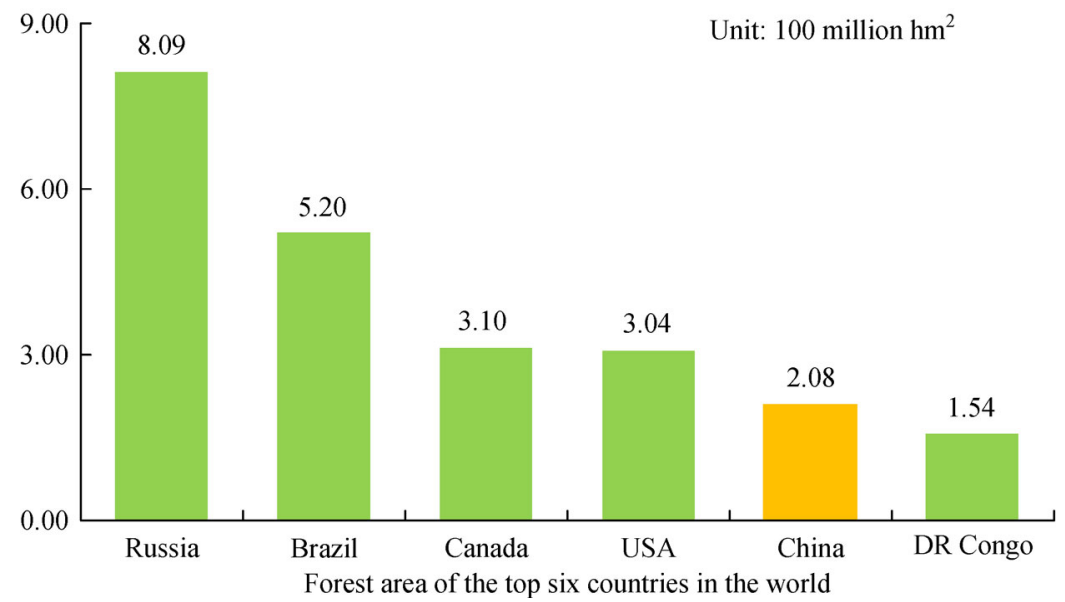

2.2 Forest stock volume of the top six countries in the world

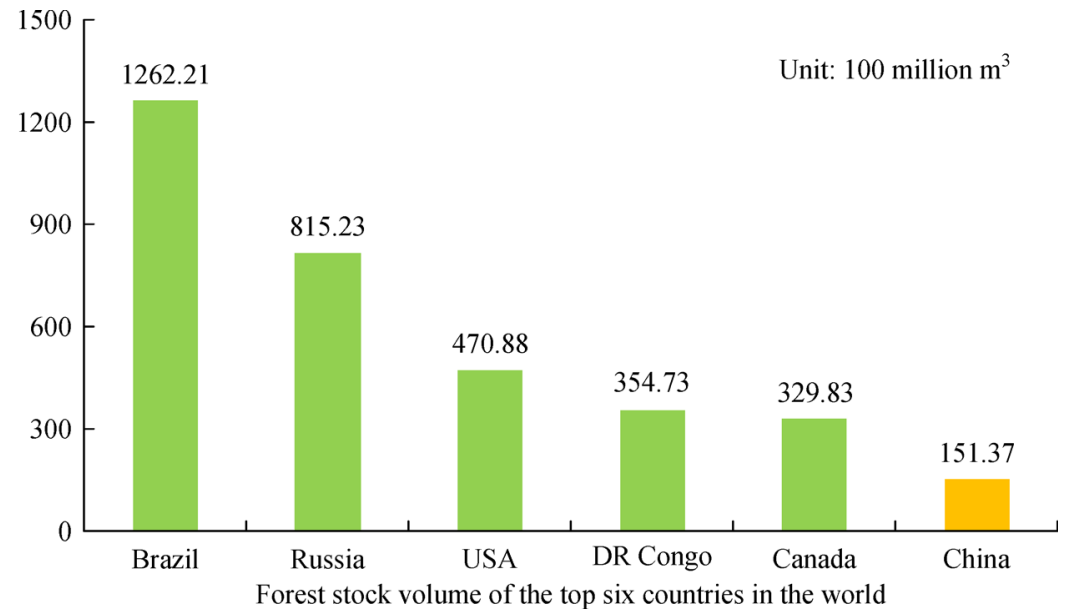




\subsection{Forest area of previous National Forest Inventories (NFIs)}

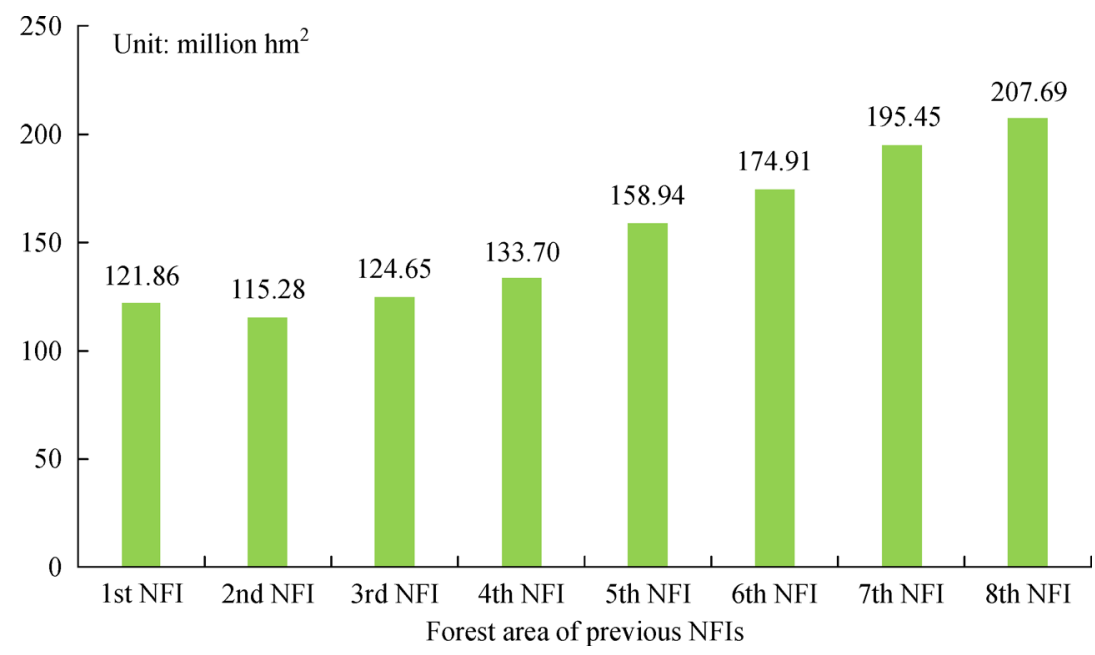

\subsection{Forest stock volume of previous NFIs}

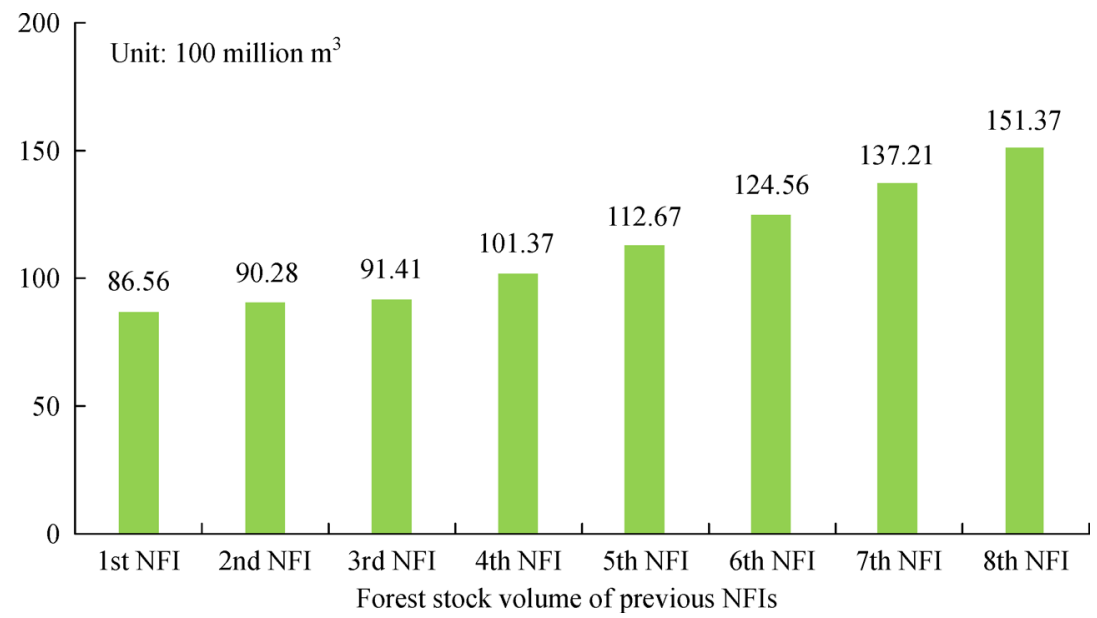

\subsection{Forest coverage of previous NFIs}

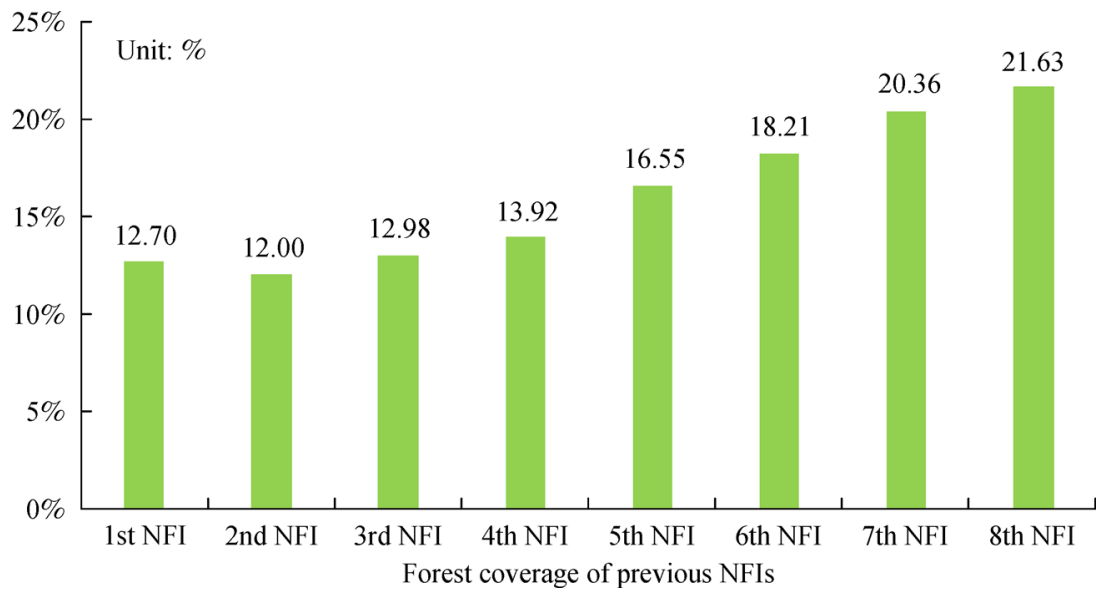




\subsection{Components of forestland area}

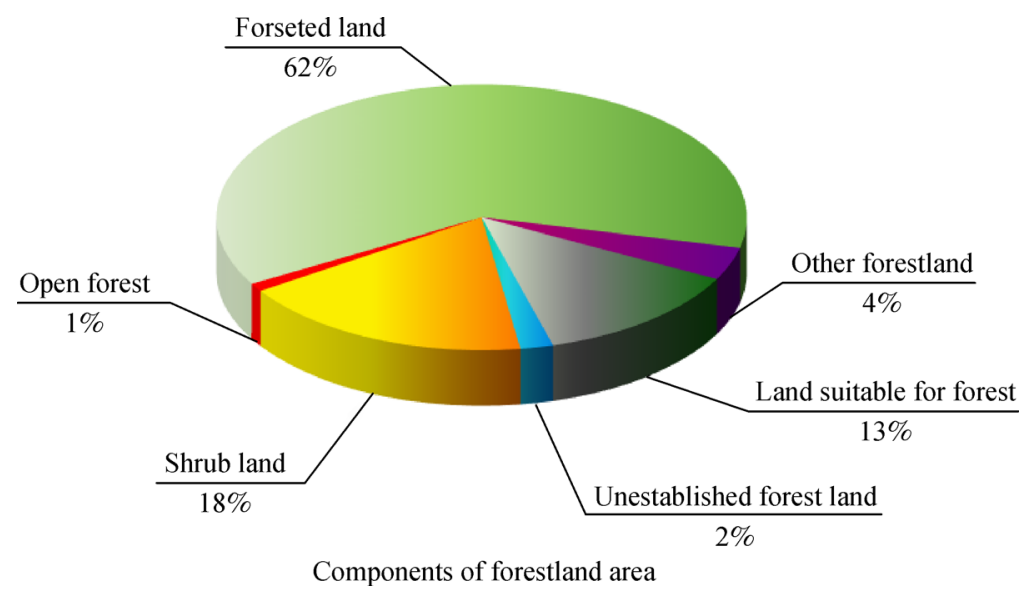

2.7 Components of forest stock volume

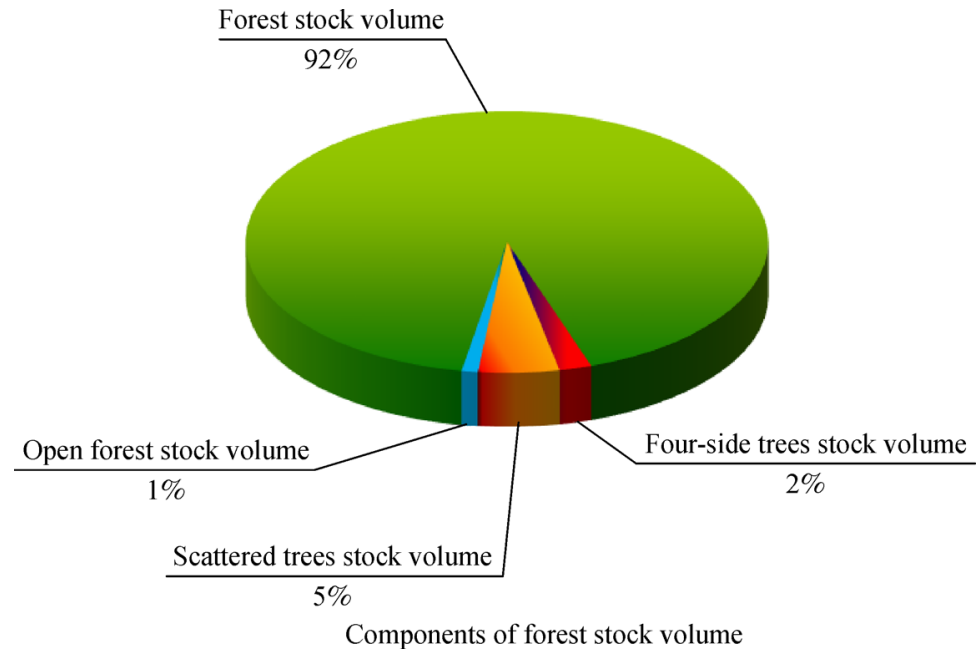

2.8 Proportion of arbor forest area and stock volume by age group

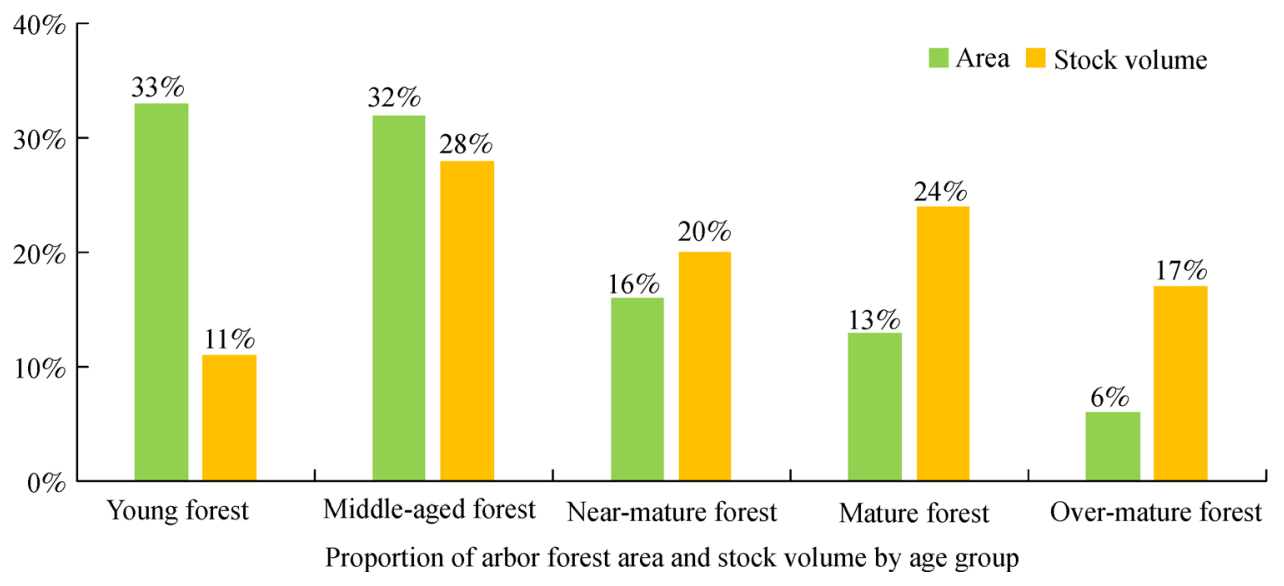




\subsection{Area and stock volume of major dominant tree species (groups) in arbor forests}

\begin{tabular}{lcccc}
\hline Dominant species (groups) & Area $\left(10000 \mathrm{hm}^{2}\right)$ & Area ratio/\% & Stock volume/(100 million $\left.\mathrm{m}^{3}\right)$ & Stock volume ratio/\% \\
\hline Quercus & 1672 & 10.15 & 12.94 & 8.76 \\
Betula & 1126 & 6.84 & 9.18 & 6.21 \\
Cunninghamia lanceolata & 1096 & 6.66 & 7.26 & 4.91 \\
Larix gmelinii & 1069 & 6.50 & 5.91 & 6.77 \\
Pinus massoniana & 1001 & 6.08 & 6.24 & 4.00 \\
Populus & 997 & 6.06 & 5.02 & 4.22 \\
Pinus yunnanensis & 455 & 2.76 & 1.60 & 3.40 \\
Eucalyptus & 446 & 2.71 & 9.99 & 1.09 \\
Picea asperata & 421 & 2.56 & 2.00 & 6.76 \\
Platycladus orientalis & 366 & 2.22 & 70.15 & 1.35 \\
Total of top 10 species (groups) & 8649 & 52.54 & & 47.47 \\
\hline
\end{tabular}

\subsection{Forest structure and functions}

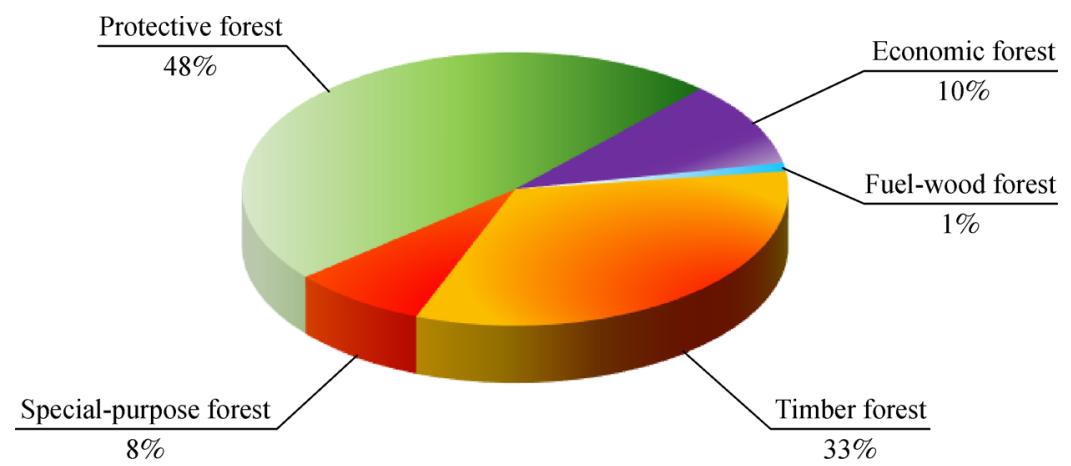

\subsection{Area and stock volume of natural arbor forest by age group}

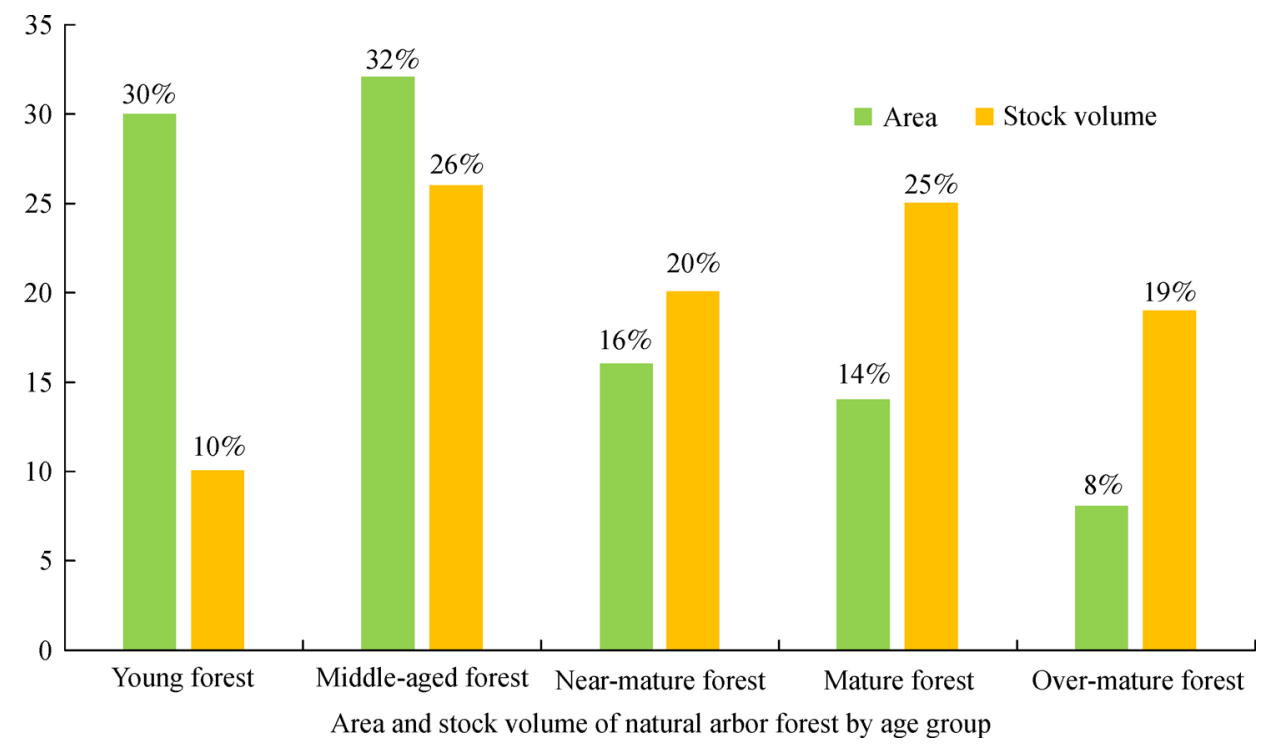




\subsection{Area and stock volume of dominant species (groups) in natural forest}

\begin{tabular}{lcccc}
\hline Dominant species (groups) & Area $\left(10000 \mathrm{hm}^{2}\right)$ & Area ratio/\% & Stock volume/(100 million $\left.\mathrm{m}^{3}\right)$ & Stock volume ratio/\% \\
\hline Quercus & 1610 & 13.70 & 12.81 & 10.42 \\
Betula & 1112 & 9.46 & 9.14 & 7.43 \\
Larix gmelinii & 756 & 6.43 & 8.17 & 6.65 \\
Pinus massoniana & 694 & 5.91 & 4.19 & 3.41 \\
Pinus yunnanensis & 410 & 3.49 & 4.77 & 3.88 \\
Picea asperata & 385 & 3.27 & 9.87 & 8.03 \\
Abies fabri & 308 & 2.62 & 11.65 & 9.47 \\
Cupressus funebris & 220 & 1.87 & 1.39 & 1.13 \\
Cunninghamia lanceolata & 202 & 1.72 & 1.01 & 0.82 \\
Pinus densata & 156 & 1.33 & 3.49 & 2.84 \\
Total of top ten species (groups) & 5853 & 48.04 & 66.49 & 54.08 \\
\hline
\end{tabular}

\subsection{Area and stock volume of arbor plantation by age group}

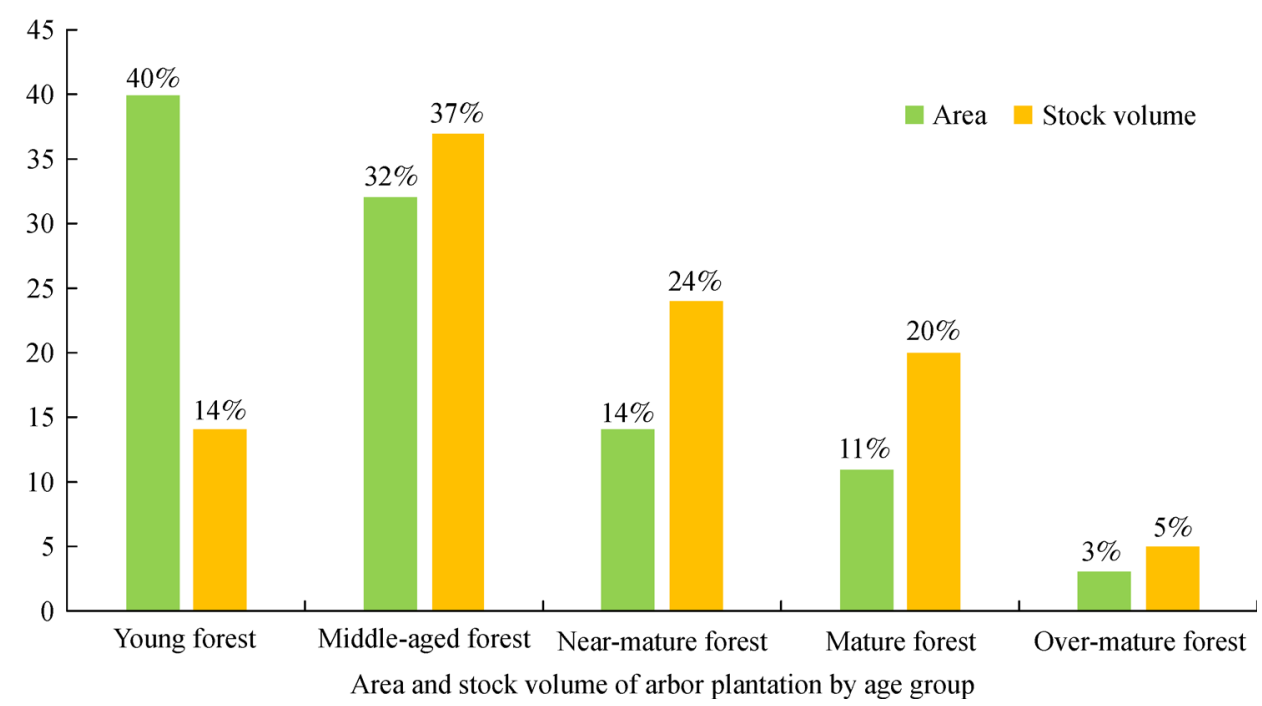

\subsection{Area and stock volume of dominant species (groups) in plantation}

\begin{tabular}{lcccc}
\hline Dominant species (groups) & Area/(10000 $\left.\mathrm{hm}^{2}\right)$ & Area ratio/\% & Stock volume/(10000 $\left.\mathrm{hm}^{2}\right)$ & Stock volume ratio/\% \\
\hline Cunninghamia lanceolata & 895 & 19.01 & 6.25 & 25.18 \\
Populus & 854 & 18.14 & 5.03 & 20.25 \\
Eucalyptus & 445 & 9.47 & 1.60 & 6.46 \\
Larix gmelinii & 314 & 6.66 & 1.84 & 7.42 \\
Pinus massoniana & 306 & 6.51 & 0.72 & 6.91 \\
Pinus tabuleaformis & 161 & 3.42 & 0.61 & 2.66 \\
Cupressus funebris & 146 & 3.11 & 0.41 & 2.46 \\
Pinus elliottii & 134 & 2.85 & 0.27 & 1.63 \\
Robinia pseudoacacia & 123 & 2.60 & 0.13 & 1.09 \\
Quercus & 61 & 1.30 & 18.52 & 0.52 \\
Total of top ten species (groups) & 3439 & 73.07 & 74.58 \\
\hline
\end{tabular}

